\title{
Evaluación de factores de riesgo como el género $y$ antigüedad del permiso de conducción en la posibilidad de provocar accidentes de tránsito
}

\author{
Evaluation of risk factors such as gender and seniority of driving license in \\ the possibility of causing traffic accidents
}

Andrés Noguera Cundar. ${ }^{1}$, Mónica Moreno Barriga. ${ }^{2}$, Fabián Bastidas Alarcon. ${ }^{3}$ \& Fabián Moreno Barriga. ${ }^{4}$

Recibido: 30-10-2018 / Revisado: 4-12-2018 /Aceptado: 22-12-2018/ Publicado: 05-01-2019

\begin{abstract}
.
DOI: https://doi.org/10.33262/cienciadigital.v3i1.296

Traffic accidents are adverse events that occur in all social areas worldwide, which apparently follow a random pattern, however, may have certain characteristics or linked characteristics, which can be identified with the help of statistical techniques. The present research work aims to find characteristics related to the driving of automobiles, in this case performing an analysis of risk factors on the influence of inexperience driving drivers, and with a differentiation or separation between the same gender, on the responsibility within a traffic accident, to achieve this purpose a set of data of road accidents was used, which after a cleaning and filtering, were subjected to an initial descriptive analysis to identify important characteristics of drivers and accidents, later statistical data mining techniques were used, such as: Random Forest to determine the importance of the variables and how they directly intervene in the responsibility of a driver in a road incident, and Classification Trees (CART) where it is observed as this technique performs a clear classification of var involved, allowing accurate and concrete information of the graph.

The results show an important difference between the participation of both sexes in accidents, in addition it is possible to appreciate the significant variation that exists in the responsibility in a road accident, it is possible to observe that the methodology used is adequate to differentiate the phenomena present during the driving. It allows
\end{abstract}

\footnotetext{
${ }^{1}$ Escuela Superior Politécnica de Chimborazo, Chimborazo, Ecuador, andres.noguera@espoch.edu.ec

${ }^{2}$ Escuela Superior Politécnica de Chimborazo, Chimborazo, Ecuador, monica.moreno@espoch.edu.ec

${ }^{3}$ Escuela Superior Politécnica de Chimborazo, Chimborazo, Ecuador, fabian.bastidas@espoch.edu.ec

${ }^{4}$ Escuela Superior Politécnica de Chimborazo, Chimborazo, Ecuador, fabian.moreno@espoch.edu.ec
} 
a classification of variables that provides clear evidence of the differences between men and women at the wheel, including the respective analysis of inexperience and how it influences the occurrence of a traffic accident.

Keywords: Evaluation, Risk Factors, Driving Permit, Traffic Accidents

\section{Resumen.}

Los accidentes de tránsito, son eventos adversos que se presentan en todo ámbito social a nivel mundial, los cuales aparentemente siguen un patrón aleatorio, sin embargo, pueden tener ciertas características o particularidades vinculadas, mismas que pueden ser identificadas con la ayuda de técnicas estadísticas.

El presente trabajo de investigación pretende encontrar características, relacionadas con la conducción de automóviles, en este caso realizando un análisis de factores de riesgo sobre la influencia de la inexperiencia al volante de los conductores, y con una diferenciación o separación entre el género de los mismos, sobre la responsabilidad dentro de un accidente de tránsito, para lograr este propósito se utilizó un conjunto de datos de siniestros viales, que después de una limpieza y filtrado, fueron sometidos a un análisis descriptivo inicial para identificar características importantes de conductores y accidentes, posterior a ello se emplearon técnicas estadísticas de minería de datos como: Random Forest para determinar la importancia de las variables y cómo estas intervienen directamente en la responsabilidad de un chofer en un incidente vial, y de Árboles de Clasificación (CART) en donde se observa como esta técnica realiza una clasificación clara de las variables implicadas, permitiendo obtener información precisa y concreta de la gráfica.

Los resultados muestran una diferencia importante entre la participación de ambos sexos en accidentes, además se puede apreciar la variación significativa que existe en la responsabilidad en un percance vial, es posible observar que la metodología utilizada es adecuada para diferenciar los fenómenos presentes durante la conducción pues permite una clasificación de variables que arroja evidencias claras de las diferencias existentes entre hombres y mujeres al volante, incluyendo el análisis respectivo de la inexperiencia y cómo influye en la ocurrencia de un accidente de tráfico.

Palabras claves: Evaluación, Factores de Riesgo, Permiso de Conducción, Accidentes de Tránsito.

\section{Introducción.}

En la actualidad, la ocurrencia de accidentes de tránsito es un fenómeno social, que sucede en cualquier lugar. Dichos eventos deben ser considerados totalmente inadmisibles, pues existen repercusiones dentro de la comunidad, en ámbitos importantes como: el económico y el humano, siendo las pérdidas materiales en vehículos, infraestructura o retenciones en el 
flujo de tráfico, y las pérdidas humanas, los efectos negativos más evidentes, respectivamente.

Tomando en cuenta este problema se han realizado diversas investigaciones en el campo de los accidentes de tránsito, en donde se ha identificado dificultades relacionadas a la escasa información disponible y que los accidentes están asociados a eventos imprevisibles y aleatorios. Sin embargo, con la ayuda del análisis estadístico se ha logrado adquirir nuevos conocimientos, y teorías, que hacen ver que los accidentes no son sucesos fortuitos, sino que por el contrario en la mayoría de casos siguen parámetros característicos de distribución, lo que quiere decir que un incidente es consecuencia de algún fallo evitable y hasta cierto punto predecible. Se ha identificado que los accidentes se definen principalmente bajo tres variables: el conductor, el vehículo y condiciones del entorno, por lo tanto, las líneas de investigación han centrado su desarrollo en el estudio de bases de datos para lograr extraer conocimientos sobre las condiciones que influyen en los datos estadísticos actuales.

Se ha revisado trabajos similares al presente, entre los cuales destacan los de Lopez G y Oña $\mathrm{J}$, quienes han utilizado árboles de clasificación CART, para identificar factores que influyen en la severidad de los accidentes de tráfico, además en un artículo adicional se utilizan tres tipos de árboles de clasificación para evaluar la exactitud de estos métodos, lográndose determinar que si existen diferencias entre los patrones que siguen los accidentes de tránsito entre hombres y mujeres. Por otra parte Adli $\mathrm{H}$ y Balbiss Al, encontraron de forma clara ciertos estándares conductuales que influyen en la forma de conducir automóviles en vías, encontrándose diferencias significativas relacionadas a la velocidad media, habilidades y actitudes que aparecen durante el tiempo de conducción, tomando en cuenta la participación económica y social, la distancia recorrida e infracciones cometidas, de cada género, finalmente autores como Massie DL, Campbell KL, Williams AF, han encontrado tasas de participación en accidentes de tráfico bastante diferenciadas por género y edades.

Con el presente trabajo se pretende conocer los accidentes de tránsito más a fondo e identificar como algunas variables, en este caso el género de los conductores y la antigüedad de su licencia de conducir o su experiencia al volante, inciden directamente en la ocurrencia y responsabilidad de un accidente de tránsito, utilizando técnicas estadísticas descriptivas y específicas, como son: Random Forest y Árboles de Clasificación CART. Los resultados se contrastaron con estudios preliminares similares, comprobándose que en efecto estas variables inciden de manera concisa en la responsabilidad dentro de un accidente de tráfico.

\section{Metodología}

Dentro del campo de la comprensión interpretación y análisis de los accidentes de tránsito, la utilización de métodos estadísticos está completamente avalada pues la estadística permite estudiar un conjunto de datos y obtener información importante de ellos, bajo esta 
concepción en este trabajo en su etapa inicial, se utilizaron algunos conceptos de la estadística tradicional para observar y tener un acercamiento preliminar con algunas características presentes en los accidentes bajo estudio, es decir se realizó un análisis descriptivo de la participación de hombres y mujeres en accidentes de tráfico y una observación de la intervención en incidentes viales en cada subconjunto de antigüedad del permiso de conducir separado por género del conductor.

Luego de este primer contacto y observación de datos, se determinó la culpabilidad o responsabilidad del conductor dentro del accidente de tránsito, para lo cual se analizó las variables correspondientes a infracciones cometidas, en donde aquel conductor que cometió alguna infracción de tránsito y como resultado de este hecho se presentó un accidente, es catalogado como responsable, por ejemplo si uno de los conductores salto una luz roja es esta infracción la que determina a esa persona como culpable.

Esta nueva variable culpabilidad se convierte entonces en el objetivo de análisis, por lo tanto se observa como ambos géneros y en cada categoría de tiempo de experiencia al volante, presentan diferencias significativas en la culpabilidad dentro de los accidentes, una vez más utilizando estadística básica, separando en porcentaje la cantidad de personas responsables de accidentes catalogadas por género y antigüedad de permiso.

La etapa siguiente consiste en utilizar la herramienta Random Forest para determinar que variables son las más importantes o influyen directamente en la culpabilidad, para ello se toma esta variable como respuesta y un conjunto de diez variables explicativas, de esta manera se determina que variables son las más influyentes sobre la variable respuesta, además de esta forma se comprueba si el género y la antigüedad del permiso de conducir son en realidad variables predominantes en la responsabilidad de un accidente de tránsito.

Finalmente se aplicaron Árboles de Clasificación CART, como etapa final, esta técnica realiza una clasificación de variables que permite apreciar de manera concreta como el género y la antigüedad del permiso influyen en la culpabilidad de un conductor en un accidente de tránsito. En este caso por lo tanto los arboles de clasificación han permitido explorar un conjunto de datos, para encontrar patrones repetitivos, tendencias o reglas que expliquen los comportamientos conductas o normas, que aparecen en un fenómeno cotidiano, en este caso los accidentes de tránsito.

En este proyecto se han utilizado árboles para el análisis de relaciones entre una variable respuesta (responsabilidad del accidente) y una serie de variables explicativas (factores concurrentes del accidente, y características de los conductores implicados). Para obtener los resultados que se pretenden encontrar se ha utilizado una base datos de accidentes de tráfico entre dos vehículos de pasajeros, con un total de 239686 accidentes es decir 479372 conductores bajo estudio. 
Con esto se busca evaluar patrones de diferenciación entre mujeres y hombres y antigüedad del permiso, aplicando las técnicas de árboles de clasificación CART.

\section{Resultados y discusión.}

Participación en accidentes por género.

La primera etapa de la investigación corresponde a un análisis de participación diferenciada de hombres y mujeres en accidentes en donde se han obtenido los siguientes resultados en base a la muestra total:

Figura 1. Participación en accidentes por género (Autor)

\section{Participación género}

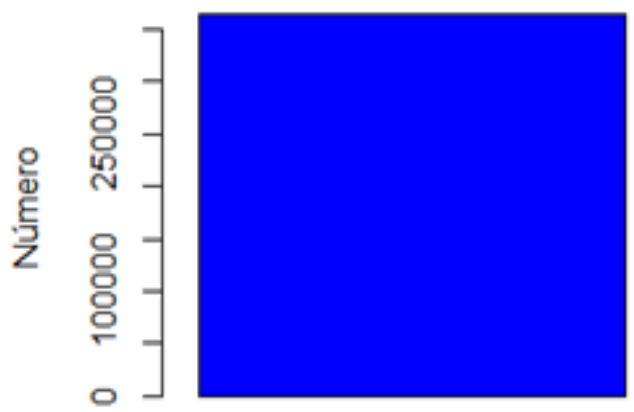

Hombre

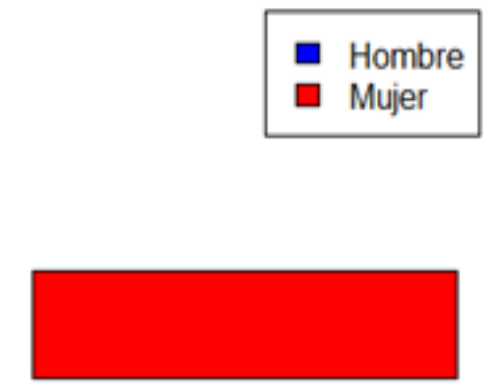

Mujer

Género

Tabla 1. Porcentaje accidentes de tráfico por género (Autor)

\begin{tabular}{lll}
\hline Género & Cantidad & \% Participación \\
\hline 1 & 364112 & $78.19 \%$ \\
0 & 103046 & $21.81 \%$ \\
\hline
\end{tabular}

Elaborado por: Grupo de Investigación

Estos resultados muestran una clara intervención superior de los hombres dentro de los accidentes de tránsito, este resultado indica una primera idea de que existen diferencias claras en las características de desempeño de ambos géneros en la conducción, por ejemplo, los hombres se encuentran presentes en más procesos diarios en los que es necesario conducir un vehículo. 


\section{Participación por género y años de conducción}

El estudio de los accidentes de tráfico relacionados con la antigüedad del permiso de conducir, es importante pues puede mostrar parámetros de comportamiento tanto en hombres y mujeres, como en cada subconjunto de tiempos de conducción, en este caso los porcentajes están calculados con respecto al total de accidentes en cada género

Figura 2. Participación en accidentes por género y años de conducción (Autor)

\section{Género-Años de conducción}

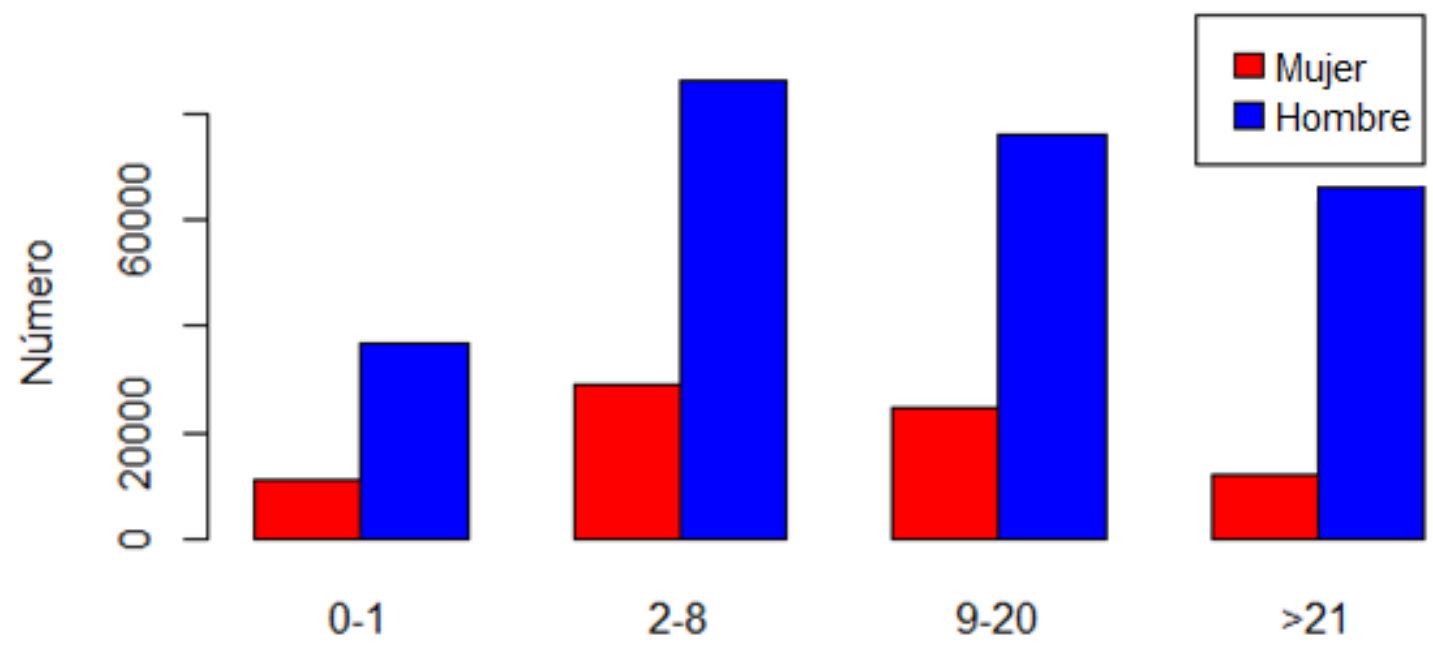

Años

Tabla 2. Porcentaje participación en accidentes por género y antigüedad del permiso (Autor)

\begin{tabular}{lllll}
\hline Años de conducción /Genero & Hombre & Cantidad & Mujer & Cantidad \\
\hline $0-1$ & $13.75 \%$ & 36740 & $14.4 \%$ & 11000 \\
$2-8$ & $32.18 \%$ & 85990 & $38.13 \%$ & 29129 \\
$9-20$ & $28.36 \%$ & 75781 & $32.01 \%$ & 24449 \\
$>21$ & $25.69 \%$ & 68632 & $15.45 \%$ & 11801 \\
\hline
\end{tabular}

Elaborado por: Grupo de Investigación

Los resultados encontrados indican que la participación en accidentes para el primer intervalo de antigüedad del permiso de conducir (0-1 año) es de $13.75 \%$ y $14.4 \%$ respectivamente para hombres y mujeres, estos valores aunque no son los más altos, presentan un porcentaje 
destacado de sucesos viales, al tener apenas un año de intervalo indican que la inexperiencia al volante es precisamente una razón en la ocurrencia de un siniestro, esto entrega una idea inicial por ejemplo de que la impericia del conductor influye en las capacidades de reacción y toma de decisiones para evitar colisiones, con la particularidad de que en este intervalo las mujeres tienen una tendencia superior, probablemente porque sus aptitudes de acción con un tiempo corto de práctica al volante sean menores.

Con este primer acercamiento a las diferencias de participación y exposición a accidentes de tráfico de varones y damas, es necesario empezar con el análisis de la responsabilidad o culpabilidad en incidentes viales en la sociedad.

\section{Determinación de responsabilidad}

Según los objetivos que persigue el presente proyecto hay que distinguir entre cada uno de los dos vehículos involucrados, si uno de sus conductores o ambos, tuvieron cierto nivel de responsabilidad en que el accidente se haya producido. Por lo tanto, la base de datos posee tres categorías de infracciones y si el conductor cometió cualquiera de los tres tipos de contravención se considera que tiene cierto nivel de responsabilidad, para ello se recodificó las variables, infracción administrativa, infracción de velocidad e infracción de conductor, dándoles únicamente valores de verdadero o falso identificando estos dos casos con los números 1 y 0 respectivamente y se realizó una pregunta condicional para obtener el resultado final, en este caso a continuación se explica un ejemplo.

Tabla 3. Descripción obtención de responsabilidad (Autor)

\begin{tabular}{|c|c|c|c|c|}
\hline $\begin{array}{l}\text { AI } \\
\text { INFVELO } \\
\text { C1 }\end{array}$ & $\begin{array}{l}\text { AJ } \\
\text { INFAD } \\
\text { M1 }\end{array}$ & $\begin{array}{l}\text { AK } \\
\text { INFCON } \\
\text { D1 }\end{array}$ & CONDICIONES & $\begin{array}{l}\text { AL } \\
\text { CULPABILID } \\
\text { AD1 }\end{array}$ \\
\hline 1 & 0 & 0 & $\begin{array}{l}\mathrm{SI}(\mathrm{AI} 2=1 ; 1 ; \mathrm{SI}(\mathrm{AJ} 2=1 ; 1 ; \mathrm{SI}(\mathrm{AK} 2= \\
1 ; 1 ; 0))) \\
\mathrm{SI}(\mathrm{AI} 3=1 ; 1 ; \mathrm{SI}(\mathrm{AJ} 3=1 ; 1 ; \mathrm{SI}(\mathrm{AK} 3=\end{array}$ & 1 \\
\hline 0 & 1 & 0 & $\begin{array}{l}1 ; 1 ; 0))) \\
\operatorname{SI}(\mathrm{AI} 4=1 ; 1 ; \operatorname{SI}(\mathrm{AJ} 4=1 ; 1 ; \operatorname{SI}(\mathrm{AK} 4=\end{array}$ & 1 \\
\hline 0 & 0 & 1 & $\begin{array}{l}1 ; 1 ; 0))) \\
\operatorname{SI}(\mathrm{AI} 5=1 ; 1 ; \operatorname{SI}(\mathrm{AJ} 5=1 ; 1 ; \mathrm{SI}(\mathrm{AK} 5=\end{array}$ & 1 \\
\hline 1 & 1 & 1 & $\begin{array}{l}1 ; 1 ; 0))) \\
\operatorname{SI}(\mathrm{AI} 6=1 ; 1 ; \operatorname{SI}(\mathrm{AJ} 6=1 ; 1 ; \operatorname{SI}(\mathrm{AK} 6=\end{array}$ & 1 \\
\hline 0 & 0 & 0 & $1 ; 1 ; 0)))$ & 0 \\
\hline
\end{tabular}

Elaborado por: Grupo de Investigación

Con esta nueva columna de responsabilidad se puede realizar un nuevo análisis estadístico de datos para intentar visualizar si existe una mayor responsabilidad en los grupos de 
antigüedad de la licencia de conducir y con la respectiva diferenciación entre hombres o mujeres dentro de la participación que tienen ambos géneros en los accidentes que se presentan en las vías.

\section{Análisis género - responsabilidad}

Como primera etapa es importante, realizar una diferenciación de la responsabilidad que tienen los conductores al ocasionar accidentes de tráfico, obteniéndose los siguientes resultados.

Figura 3. Responsabilidad por género (Autor)

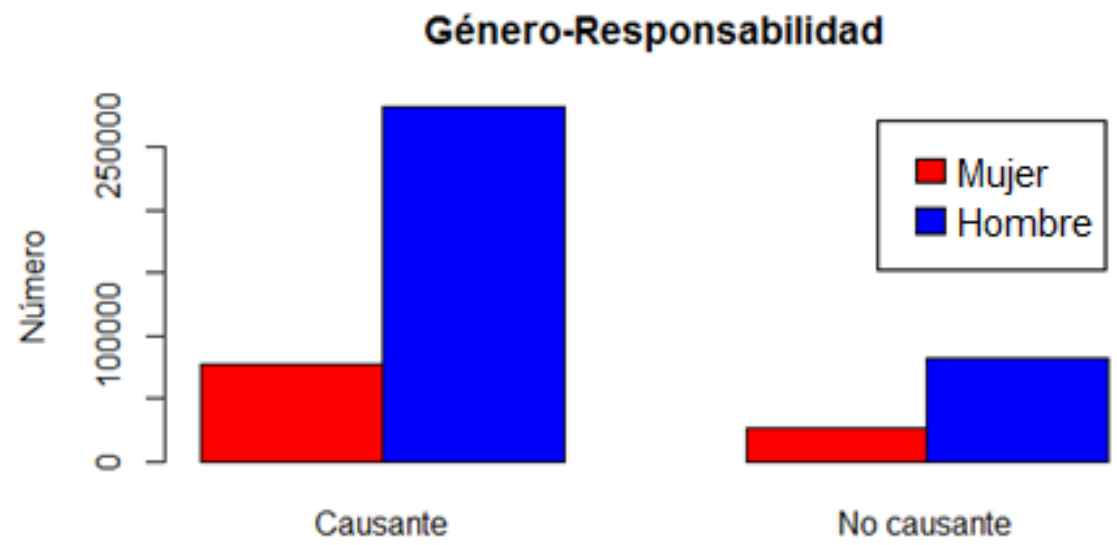

Responsabilidad

Tabla 4. Porcentaje responsabilidad por género (Autor)

\begin{tabular}{lllll}
\hline $\begin{array}{c}\text { Responsabilidad/ } \\
\text { Genero }\end{array}$ & Hombre & Cantidad & Mujer & Cantidad \\
\hline Causante & $77.26 \%$ & 281316 & $74.9 \%$ & 77183 \\
No causante & $22.73 \%$ & 82796 & $25.09 \%$ & 25863 \\
\hline
\end{tabular}

Elaborado por: Grupo de Investigación

Los valores encontrados en términos de porcentaje son calculados con relación al valor total de conductores de cada género. Los resultados muestran que los varones son quienes tienen un más alto nivel de responsabilidad, en este caso con $77.26 \%$ de hombres causantes frente a un $74.9 \%$ de mujeres responsables del choque, aunque este porcentaje no muestra una diferencia importante es esencial indicar que este hecho se debe a que la culpabilidad está calculada en base a las infracciones cometidas, y que en ciertos casos en un mismo accidente ambos conductores pudieron haber cometido infracciones, por lo tanto existiría la necesidad 
de hacer un análisis más profundo o buscar una alternativa más eficaz que permitan indicar con más precisión el culpable en un siniestro.

\section{Responsabilidad por años de conducción}

Figura 4. Responsabilidad por años de conducción (Autor)

\section{Responsabilidad-Años de conducción}

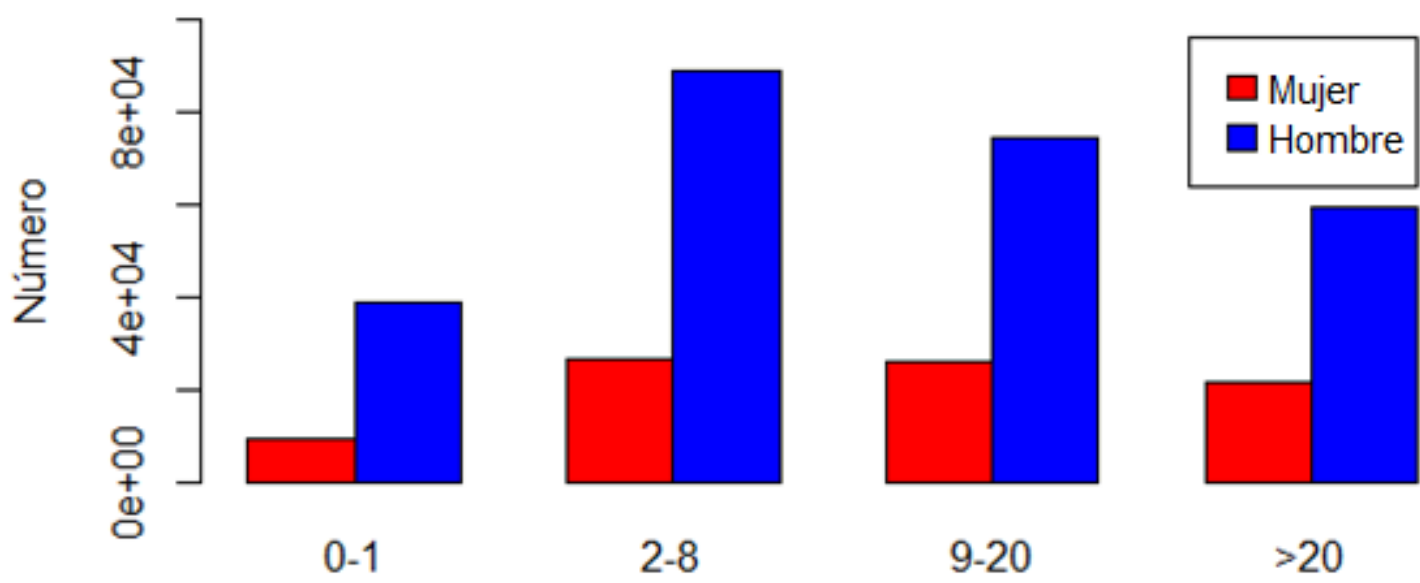

Años

Tabla 5. Porcentaje responsabilidad por género y antigüedad del permiso (Autor)

\begin{tabular}{lcllll}
\hline $\begin{array}{c}\text { Antiguiedad } \\
\text { permiso/Responsabilidad }\end{array}$ & Culpable & Cantidad & $\begin{array}{c}\text { No } \\
\text { culpable }\end{array}$ & Cantidad \\
\hline $0-1$ & $14.82 \%$ & 38731 & $11.01 \%$ & 9181 \\
$2-8$ & $34.00 \%$ & 88857 & $31.97 \%$ & 26644 \\
$9-20$ & $28.43 \%$ & 74307 & $31.47 \%$ & 26226 \\
$>20$ & $22.73 \%$ & 59406 & $25.53 \%$ & 21277 \\
\hline
\end{tabular}

Elaborado por: Grupo de Investigación

Al aplicar un análisis al grupo de culpabilidad se puede determinar que en años iniciales en tiempo de conducción (0-1 año) existe una probabilidad alta de ser responsables en un accidente, pues influyen sobre el conductor; su poca experiencia al volante, su bajo nivel de 
concentración y baja capacidad de evaluar el peligro y tomar decisiones acertadas frente a una situación que comprometa la seguridad propia de un conductor.

\section{Importancia de las variables.}

Como primer paso es necesario definir y observar si el sexo del conductor y su experiencia al volante influyen directamente sobre la culpabilidad en un accidente de tránsito, en este caso se utilizan un conjunto de variables, que nos muestran la importancia en los incidentes de tráfico.

Figura 5. Importancia de variables (Autor)

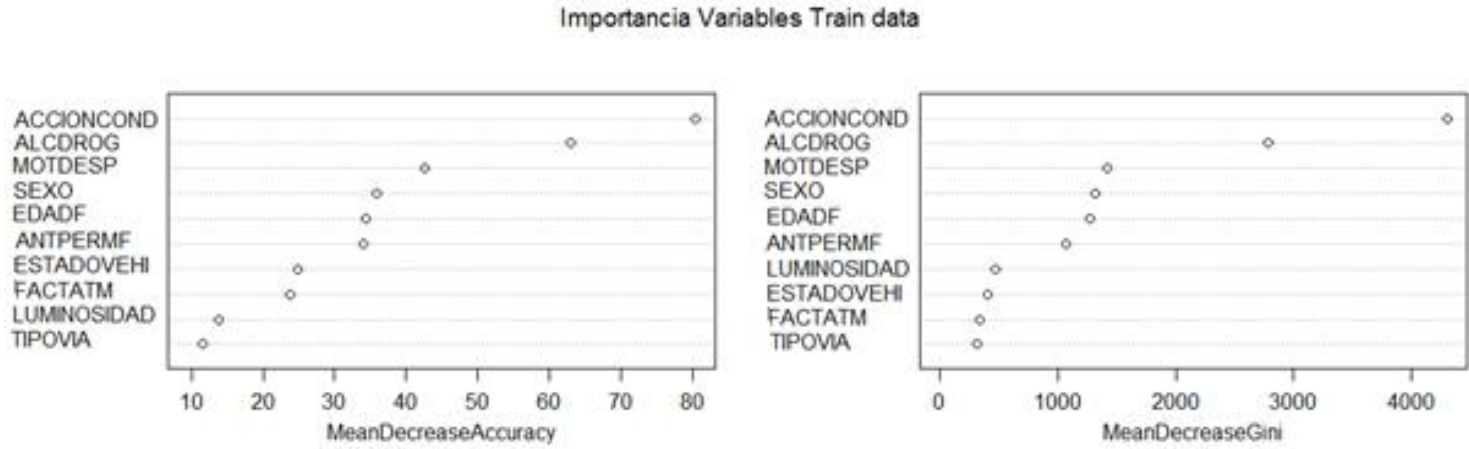

Como se puede apreciar los gráficos de MDA y MDG, son coherentes pues la importancia de las variables en el modelo en ambos casos se muestra prácticamente iguales, con variantes poco importantes en su orden, estas medidas (MDA y MDG), muestran como cada variable contribuirá a una correcta categorización en un árbol de clasificación permitiendo homogeneidad en los nodos.

A continuación, se presentan los porcentajes para cada uno de los casos.

Tabla 6. Importancia de variables, culpabilidad-Train Data (Autor)

\begin{tabular}{llllll}
\hline VAR & $\begin{array}{l}\text { ACCIONCO } \\
\text { ND }\end{array}$ & ALCDROG & MOTDESP & SEXO & EDADF \\
\hline$\%$ & 80.36 & 62.91 & 43.2 & 35.54 & 34.28 \\
\hline
\end{tabular}

\begin{tabular}{llllll}
\hline VAR & ANTPERMF & $\begin{array}{l}\text { ESTADOVE } \\
\text { HI }\end{array}$ & $\begin{array}{l}\text { FACTAT } \\
\text { M }\end{array}$ & $\begin{array}{l}\text { LUMINOSID } \\
\text { AD }\end{array}$ & $\begin{array}{l}\text { TIPOVI } \\
\text { A }\end{array}$ \\
\hline$\%$ & 32.40 & 25.32 & 24.56 & 14.03 & 13.54 \\
\hline
\end{tabular}


Elaborado por: Grupo de Investigación

Por lo tanto, los valores muestran que el modelo es adecuado y que el tratamiento de datos ha sido correcto. Árbol de clasificación.

Figura 7. Árbol de clasificación, culpabilidad por sexo y antigüedad del permiso de conducir (Autor)

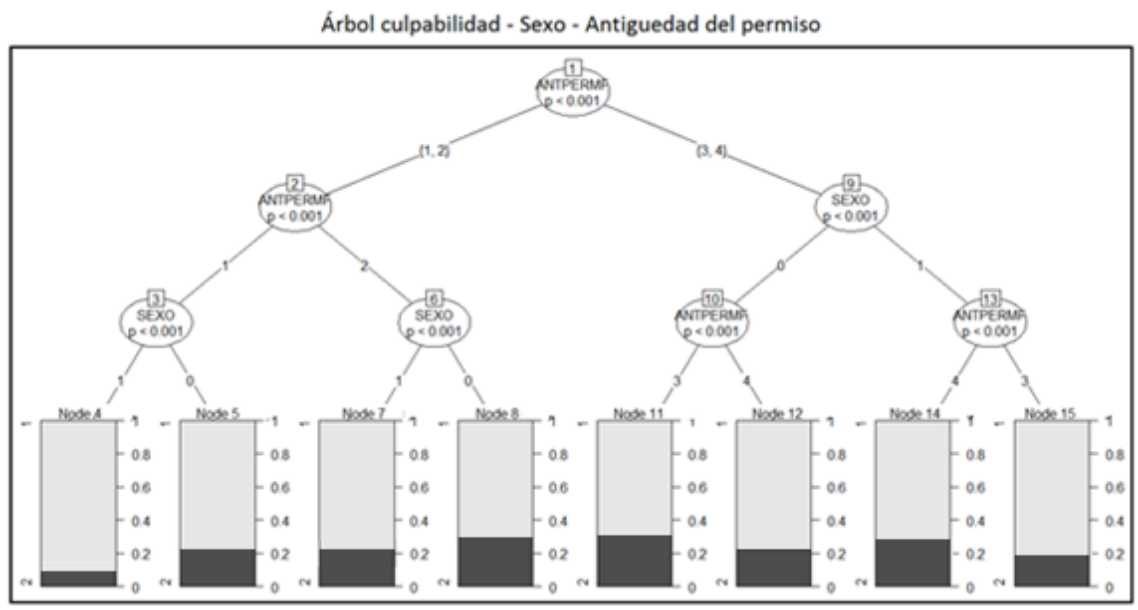

Se presenta a continuación un resumen explicativo de los resultados que muestra la gráfica anterior. Los nodos finales muestran el porcentaje de responsabilidad de los conductores en accidentes de tránsito. Con el número 1 en color claro porcentaje de responsabilidad y con número 2 en color obscuro porcentaje de no responsabilidad.

Los nodos resultantes (4) y (5) hombres y mujeres respectivamente provienen del ramal $\{1\}$ que indica un máximo de un año en la antigüedad del permiso del conductor, es decir que quienes están en su primer año de experiencia aparecen con mayor probabilidad como responsables con porcentajes de $91 \%$ y $79 \%$ respectivamente, esto debido a su inexperiencia, falta de practica e inmadurez al volante.

Los siguientes nodos respuesta (7) y (8), presentan porcentajes de responsabilidad de $78 \%$ y $67 \%$ para los sexos masculino y femenino correspondientemente, para 2 a 8 años de vigencia de la licencia de conducir, mejorando significativamente los valores debido a que las aptitudes y experiencia al volante van mejorando con el pasar de los años.

En los nodos (11) y (12) aparecen mujeres con antigüedad de permiso en los intervalos de nueve a veinte años y más de 21 años como culpables en el $77 \%$ y $78 \%$ de casos.

Y como punto final en los nodos (15) y (14) los hombres aparecen en un 79\% y $81 \%$ de probabilidad de responsabilidad en accidentes, con permisos de conducción de nueve a veinte años y más de 21 años de antigüedad. 
Se muestra a continuación una tabla resumen, en donde el valor numérico muestra el porcentaje en que cada género aparece como culpable para cada intervalo en la antigüedad del permiso de conducir.

Tabla 7. Resumen culpabilidad (Autor)

\begin{tabular}{lll}
\hline $\begin{array}{l}\text { Antigüedad } \\
\text { permiso/Responsabilidad }\end{array}$ & Hombre & Mujer \\
\hline $0-1$ & $91 \%$ & $79 \%$ \\
$2-8$ & $79 \%$ & $72 \%$ \\
$9-20$ & $76 \%$ & $69 \%$ \\
$>20$ & $81 \%$ & $78 \%$ \\
\hline
\end{tabular}

Elaborado por: Grupo de Investigación

Estos resultados permiten visualizar que durante el primer año de conducción, existe un alto índice de culpa o responsabilidad en los accidentes, luego una reducción considerable en el siguiente intervalo para el tercer rango nuevamente se mira una ligera disminución, y finalmente aumenta el porcentaje debido que en para más de 21 años antigüedad del permiso es probable que existan en la base de datos conductores de avanzada edad que también tienen propensión a accidentarse debido a sus condiciones físicas, esto sin embargo sin sobrepasa el margen del primer año de emisión de la licencia.

Se encuentran por lo tanto las siguientes situaciones importantes que explican la culpabilidad de un accidente:

- La antigüedad del permiso, es un factor determinante.

- Dentro de este tiempo transcurrido desde la emisión del permiso, el siguiente factor determinante es el género del conductor.

Además, se logra observar que la proporción de culpables es alta en los siguientes escenarios:

- Hasta 1 año de antigüedad de permiso Hombres y Mujeres $\rightarrow$ conductores noveles.

\section{Conclusiones.}

- Los conductores de ambos géneros presentan importantes diferencias en su participación y responsabilidad en accidentes de tránsito, por lo tanto, el sexo del conductor es una variable relevante.

- En un incidente vial, concurren varios factores que desencadenan la ocurrencia del 
mismo, entonces el presente estudio indica que la antigüedad del permiso de conducir influye considerablemente en que suceda un accidente pues esta variable guarda una importante relación con el grado de experiencia del conductor.

- La estadística es una herramienta adecuada que permite reconocer e interpretar ciertos fenómenos que se pueden encontrar durante la conducción y de ser el caso durante un accidente vial, pues muestra datos de una manera precisa y comprensible de tal forma que se logren comprobar hipótesis planteadas.

\section{Referencias bibliográficas.}

Al-Balbissi, A. H. (15 de Septiembre de 2010). Role of gender in road accidents. Traffic Injury Prevention.

Bose, D., Gomez, M. S., \& Crandall, J. R. (Diciembre de 2011). Vulnerability of female drivers involved in motor vehicle crashes: an analysis of Us population at risk. APHA.

de Oña , J., López, G., \& Abellán, J. (6 de Marzo de 2012). Extracting decision rules from police reports through decision trees. ELSEVIER.

Gray, R.C., Quddus, M.A., Evans, A., (2008). "Injury severity analysis of accidents involving young male drivers in Great Britain".Journal of Safety Research 39, 483-495.

Pakgohar, A., Tabrizi, R.S., Khalilli, M., Esmaeili, A., (2010). The role of human factor in incidence and severity of road crashes based on the CART and LR regression: a data mining approach. Procedia Computer Science 3, 764-769.

Montella, A., Aria, M., D’Ambrosio, A.,Mauriello, F., 2011. Analysis of powered twowheeler crashes in Italy by classification trees and rules discovery. Accident Analysis and Prevention, in press.

Williams AF. Teenage drivers: patterns of risk. J Safety Res 2003;34:5-15.

Catchpole JE, MacDonald WA, Bowland L. Young driver research program: The influence of age-related and experience-related factors on reported driving behaviour and crashes. Canberra: Federal Office of Road Safety. Contract Report 143.

Braitman, K. A., Kirley, B. B., McCartt, A. T., \& Chaudhary, N. K. (2008). Crashes of novice teenage drivers: Characteristics and contributing factors. Journal of Safety Research, $39(1), 47-54$.

Williams, A. F., Ferguson, S. A., \& McCartt, A. T. (2007). Passenger effects on teenage driving and opportunities for reducing the risks of such travel. Journal of Safety Research, 38(4), 381-390.

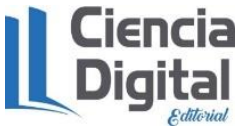


Para citar el artículo indexado.

Noguera A., Moreno M., Bastidas F. \& Moreno F. (2019). Evaluación de factores de riesgo como el género y antigüedad del permiso de conducción en la posibilidad de provocar accidentes de tránsito. Revista electrónica Ciencia Digital 3(1), 351-364. Recuperado desde: http://cienciadigital.org/revistacienciadigital2/index.php/CienciaDigital/article/view/296/70 $\underline{6}$

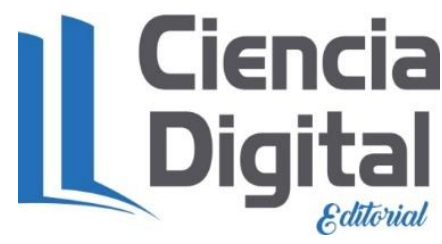

El artículo que se publica es de exclusiva responsabilidad de los autores y no necesariamente reflejan el pensamiento de la Revista Ciencia Digital.

El artículo queda en propiedad de la revista y, por tanto, su publicación parcial y/o total en otro medio tiene que ser autorizado por el director de la Revista Ciencia Digital.
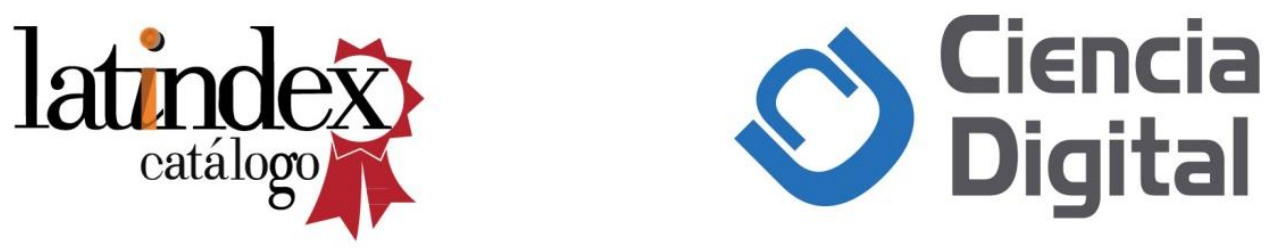